\title{
Homeostasis: A tensile state
}

\author{
N. Podewitz ${ }^{1}$, M. Delarue ${ }^{2}$ and J. Elgeti ${ }^{1}$ \\ 1 Theoretical Soft Matter and Biophysics, Institute of Complex Systems, Forschungszentrum Juelich - 52425 Juelich, \\ Germany \\ 2 Institut Curie, VMR 168, 5 rue Piere et Marie Curie, 75005 Paris, France
}

\begin{abstract}
PACS 87.19.R- - Mechanical and electrical properties of tissues and organs
PACS 87.17.Aa-Modeling, computer simulation of cell processes

PACS 87.17. Ee - Growth and division
\end{abstract}

\begin{abstract}
Mechanics play a significant role during tissue development. One of the key characteristics that underlies this mechanical role is the homeostatic pressure, which is the pressure stalling growth. In this work, we explore the possibility of a negative bulk homeostatic pressure by means of a mesoscale simulation approach and experimental data of several cell lines. We show how different cell properties change the bulk homeostatic pressure, which could explain the benefit of some observed morphological changes during cancer progression. Furthermore, we study the dependence of growth on pressure and estimate the bulk homeostatic pressure of five cell lines. Four out of five result in a bulk homeostatic pressure in the order of minus one or two kPa.
\end{abstract}

Introduction. - While growth of eukaryotic cells is mainly determined by signaling and nutrition, the notion that mechanics also play a role has been advancing continuously over the years. Ingber and colleagues noted already thirty years ago that tensile stresses can regulate tissue architecture [1]. Today, mechanotransduction and mechanobiology are important fields of research $[2,3]$. From a physics point of view, a cell has to increase its volume in order to accommodate for new cell material. In terms of thermodynamics, pressure is the conjugated variable of volume. Therefore, it seems natural to investigate the pressure cells can develop as well as its feedback onto growth. Mechanical feedback on growth has been implemented in many different ways $[4,5]$. One intuitive approach is to expand the growth rate in powers of the pressure around the zero growth rate pressure - the homeostatic pressure [6]. In this theory, the homeostatic state of a tissue is characterized by the balance of cell division and cell death (apoptosis) rate, which reflects a dynamic steady state with respect to the number of cells. The tissue maintains a well defined pressure, called homeostatic pressure $P_{H}$, and the overall growth rate $k$ around the homeostatic state depends on the difference between $P_{H}$ and the externally imposed pressure $P^{i}$ :

$$
k=\kappa\left(P_{H}-P^{i}\right)
$$

The concept is best understood with a simple gedankenexperiment: a tissue is confined in a small chamber, where one of the walls acts as a movable piston connected to a spring. Cells are placed inside the chamber and the tissue grows, compressing the spring. Eventually, the force exerted by the piston on the cells is large enough to slow down division, yielding a steady state, where apoptosis balances cell division.

It is a challenging task to measure this homeostatic pressure experimentally. In some attempts, researchers embedded cells in an elastic shell that can be deformed by the tissue, but the tissue never reached a steady-state and therefore a well-defined pressure [7]. Another approach exerted a compressive stress directly onto multicellular spheroids $[5,8,9]$, which resulted in a slow down in growth.

A detailed analysis showed that spheroid growth is highly dominated by surface effects in the sense that cell division happens mainly at the first 2-3 cell layers of the free surface. Although some physiological gradients of nutrients or growth factors exist [10], this surface growth effect is also seen in simulations [9], where we can decouple the mechanics from the biochemistry. A possible intuitive explanation by simple mechanics would be as follows. A growing cell increases its volume, creating a strain dipole. The energy required to insert such a dipole at the surface of the tissue is smaller than in the bulk as in the former case part of the necessary strain field is cut away. There- 


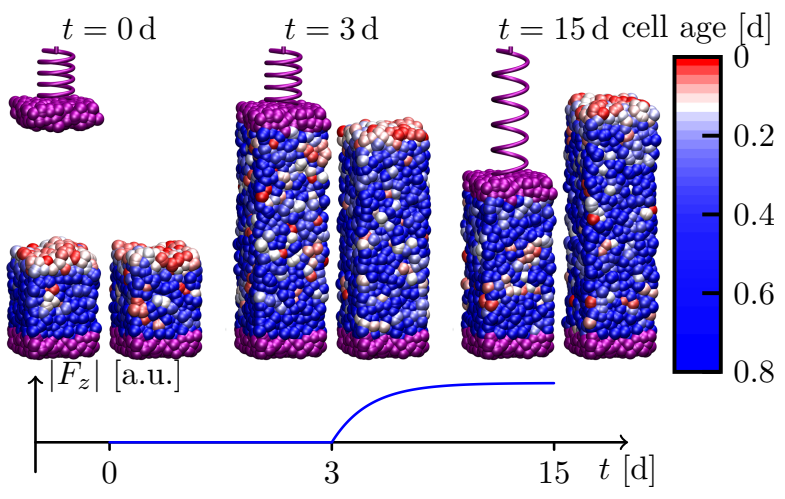

Fig. 1: Visualization of the concept of a negative homeostatic pressure. Sticky walls are modeled by particles similar to normal cells, except they are fixed with a strong harmonic potential (purple particles). The piston at the top is, furthermore, subjected to a weak harmonic potential depicted by the spring. Snapshots at different times $t$ are shown for the same simulation with (left) and without (right) piston (see Supplementary Movie S1). Cells are color coded according to their age (red corresponds to recently divided cells, while blue corresponds to cells that did not divide for a certain amount of time). Note that most cell divisions happen at free surfaces. The sticky piston cuts away this free surface and is pulled down due to the negative bulk homeostatic pressure.

fore, division is favored at free surfaces.

The net growth in the bulk of the aforementioned tissue experiments was determined to be negative. This leads to a dynamic steady state, where the apoptotic core is balanced by an influx of cells from the surface [11]. Could we thus deduce the homeostatic pressure to be negative? Does a negative homeostatic pressure even make sense?

We used the same mesoscopic simulation model as in $[8,9,12]$ to perform a gedankenexperiment of tissue growth in a box with a piston (see Fig. 1 and Supplementary Movie S1), demonstrating the concept and stability of a negative homeostatic pressure. Initially, cells are seeded at the bottom of the box, adhere to the wall and start to grow upwards due to the free surface. The piston, acting as a sticky surface, however, cuts away this free surface and leaves only the on-average apoptotic bulk. The resulting decrease in cell number and the adhesion forces between cells pull down the piston. Stress in the bulk becomes tensile, and the growth rate increases until a different steady state (under tension) is reached.

Methods. - In order to study the behavior of growing tissues, we use the same mesoscopic simulation model as in $[8,9,12]$. Briefly, each cell consists of two point particles that repel each other with a growth force $\boldsymbol{F}_{i j}^{g}=$ $B /\left(r_{i j}+r_{0}\right)^{2} \hat{\boldsymbol{r}}_{\boldsymbol{i} \boldsymbol{j}}$, where $B$ is the growth force strength, $r_{i j}$ the distance between the particles $i$ and $j, r_{0}$ some constant and $\hat{\boldsymbol{r}}_{\boldsymbol{i} \boldsymbol{j}}$ the unit displacement between $i$ and $j$. Upon exceeding a certain critical distance, the cell divides. Apoptosis is introduced as a constant probability for each cell to disappear. These two are the only active components in the simulations. The volume exclusion force $\boldsymbol{F}_{i j}^{v}=f_{0}\left(R_{p p}^{5} / r_{i j}^{5}-1\right) \hat{\boldsymbol{r}}_{\boldsymbol{i j}}$ ensures impenetrability of the cells with the cell-cell potential coefficient $f_{0}$. All interactions are short ranged with a cut-off radius $R_{p p}$, beyond which all interaction are set to zero. Cell adhesion is represented by a simple constant force $\boldsymbol{F}_{i j}^{a}=-f_{1} \hat{\boldsymbol{r}}_{\boldsymbol{i} \boldsymbol{j}}$ and tuned by the adhesion strength $f_{1}$.

To account for dissipation and random fluctuations, we use a dissipative particle dynamics (DPD) type thermostat. It consists of a random force $\boldsymbol{F}_{i j}^{r}=\sigma \omega^{R}\left(r_{i j}\right) \xi_{i j} \hat{\boldsymbol{r}}_{\boldsymbol{i j}}$ with $\sigma$, the strength of this force, $\xi_{i j}=\xi_{j i}$ a symmetric gaussian random variable with zero mean and unit variance and a weight function $\omega^{R}\left(r_{i j}\right)$ and a dissipative force $\boldsymbol{F}_{i j}^{d}=-\gamma \omega^{D}\left(r_{i j}\right)\left(\boldsymbol{v}_{i j} \cdot \hat{\boldsymbol{r}}_{\boldsymbol{i j}}\right) \hat{\boldsymbol{r}}_{\boldsymbol{i j}}$ with $\gamma$ the strength of this force, $\boldsymbol{v}_{i j}=\boldsymbol{v}_{j}-\boldsymbol{v}_{i}$, the relative velocity between $i$ and $j$ and a weight function $\omega^{D}\left(r_{i j}\right)$. Unless explicitly mentioned, we use the same standard parameter set and notation as in $[8,9]$, i.e. all quantities denoted with a ${ }^{*}$ are in terms of the standard parameter set (e.g. $\left.B^{*}=B / B_{\text {ref }}\right)$.

To model different compressibilities, the volume exclusion force prefactor $f_{0}^{*}$ and the adhesion force prefactor $f_{1}^{*}$ are varied simultaneously, while $f_{0}^{*} / f_{1}^{*}$ is kept constant. Thus, the minimum of the potential between particles is fixed, whereas the second derivative of the potential around this minimum is varied, leading to softer/stiffer tissues. The compressibility $K$ is defined as $1 / f_{0}^{*}$.

In order to measure the bulk dependencies on pressure, we implement a constant pressure ensemble as detailed in [13]. The described method imposes a defined pressure on a system with full periodic boundaries. This pressure is maintained through a periodic rescaling of the box volume by a factor

$$
\chi=1-\beta_{T} \frac{\Delta t}{t_{P}}\left(P-P^{i}\right),
$$

where $\beta_{T}$ is the isothermal compressibility, $\Delta t$ the simulation time step, $t_{p}$ a relaxation time constant and $P^{\mathrm{i}}$ the pressure we impose on the system (in simulations: $\beta_{T} / t_{P}=1 \mathrm{~d}^{-1} \mathrm{kPa}^{-1}$ with a time step of $\Delta t=10^{-3} \mathrm{~d}$ ). Furthermore, the center of mass of all cells is rescaled by $\sqrt[3]{\chi}$. There are in general no constraints on the imposed pressure, which makes it possible to simulate systems under tension $\left(P^{i}<0\right)$ as well. Following [14], the current pressure $P$ inside the full periodic simulation box is calculated through the evaluation of the virial stress

$$
\sigma_{\alpha \beta}=-\frac{1}{V}\left[\sum_{i} m_{i} v_{\alpha}^{i} v_{\beta}^{i}+\sum_{i, j} r_{\alpha}^{i j} f_{\beta}^{i j}\right],
$$

where $\sum_{i}$ is the sum over all particles, $v_{\alpha}^{i}$ the $\alpha$ th component of the velocity of particle $i, \sum_{i, j}$ the sum over all pair interactions that act on $i, r_{\alpha}^{i j}=r_{\alpha}^{i}-r_{\alpha}^{j}$ the $\alpha$ th component of the distance vector between $i$ and $j$ and $f_{\beta}^{i j}$ the $\beta$ th component of the force acting on particle $i$. We define the mean pressure as $P=-1 / 3 \operatorname{Tr} \sigma_{\alpha \beta}$. Without rescaling, the 
system reaches its homeostatic state, where the measured pressure equals the homeostatic pressure.

Experimentally, pressure is applied to spheroids by adding a biocompatible polymer, Dextran, to the culture medium. Dextran does not penetrate the spheroid, and rather exert an osmotic stress onto the whole structure. This method, however, can only apply compressive stresses onto the surface of a growing spheroid. The effect of Dextran can be mimicked in the simulations by a purely repulsive van der Waals gas, which then applies the stress on the system $[8,9,11]$. This method, however, requires a complete tissue spheroid placed in a simulation box with hard walls. The pressure applied on the spheroid due to the additional gas particles is then calculated from the momentum transfer onto the walls. A direct comparison of the results of the constant pressure ensemble and the van der Waals gas show a perfect agreement. However, the constant pressure ensemble is computationally much more efficient (due to its smaller system size) and additionally can also impose tensile stresses onto the system. Therefore, all bulk growth rate related results shown in this article, were obtained with this method.

The experimental data consists of 5 distinct cell lines of immortalized cells. The CT26 and HT29 cell lines both come from colon carcinoma (HT29 originate from a human tumor and have a more epithelial phenotype, while CT26 originate from a murine tumor and have a mesenchymal phenotype). The AB6 cell line comes from the immortalization of murine spontaneous sarcoma and also have a mesenchymal phenotype. The FHI cell line, coming from Schwannoma, and the BC52 cell line, coming from human breast cancer, both have an epithelial phenotype.

Results. - With the gedankenexperiment described above, we have seen how a negative homeostatic pressure could still form a macroscopic tissue and develop a tensile state (See Fig. 1). However, to quantify the stress repsonse of the tissue this setup might still contain boundary effects. We thus utilize the constant pressure ensemble to better understand the response of growing tissues to mechanical stresses and describe experimental data in more detail. Starting from the standard parameter set as defined in $[8,9]$, we investigated the bulk homeostatic pressure dependence on different model parameters. We focused on the growth force strength $B^{*}$, the adhesion strength $f_{1}^{*}$ and the cell stiffness $K$, since these parameters have a straightforward analogon in real tissues and can in principle be measured [15-23]. The results are shown in Fig. 2 and display a linear relationship between $P_{H}^{b}$ and $f_{1}^{*}$ and $B^{*}$, respectively. The critical adhesion strength $f_{c}^{*}\left(B^{*}\right)$ characterizes the transition from a negative to a positive $P_{H}^{b}$. Finally, we find $P_{H}^{b}$ to increase with the compressibility $K$ (see Fig. 2b). This increase is somewhat nonlinear and the slope changes with $B^{*}$.

A negative $P_{H}^{b}$ naturally leads to a stable steady state for a freely growing tissue. Assuming a similar two rate growth model as in [8], the time evolution of the radius $R$ a)

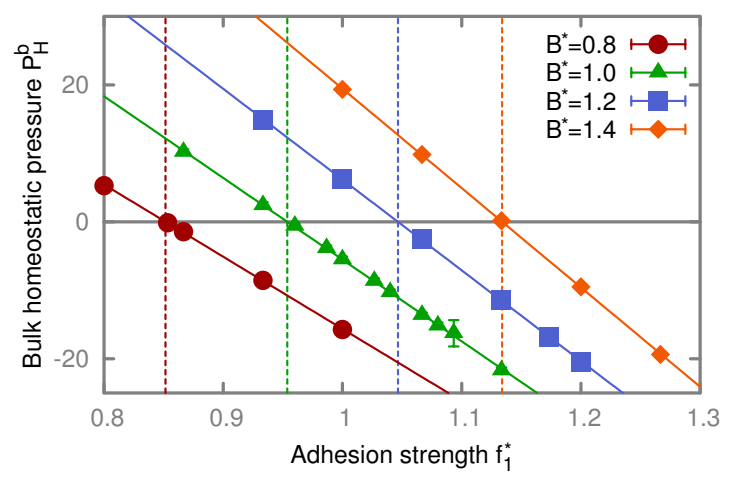

b)

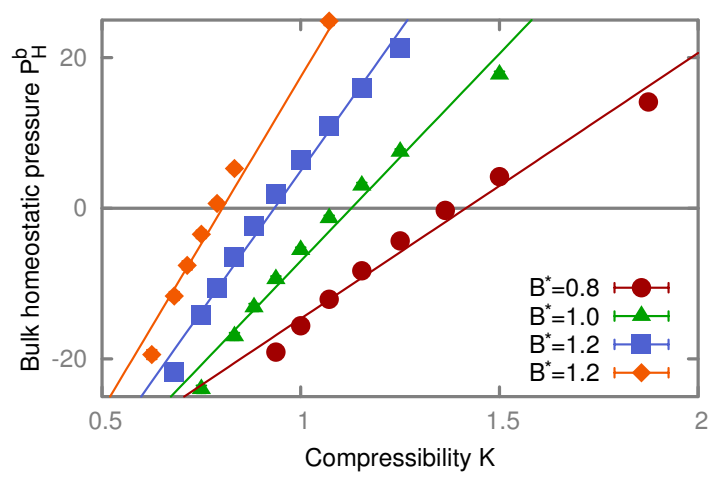

Fig. 2: Bulk homoestatic pressure dependence on model parameters. a) Bulk homeostatic pressure $P_{H}^{b}$ in simulation units as a function of adhesion strength $f_{1}^{*}$ and growth force $B^{*}$. Solid lines represent linear fits. b) Bulk homeostatic pressure $P_{H}^{b}$ as a function of cell compressibility $K$. Solid lines represent linear fits. All data were measured in full periodic boundaries. Error bars represent standard deviations.

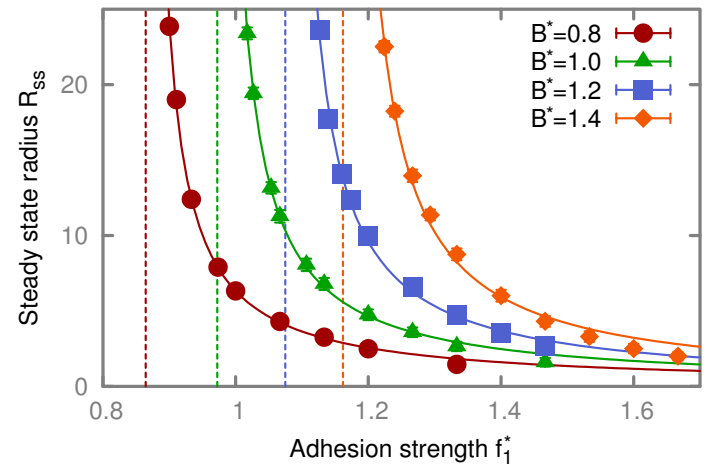

Fig. 3: Steady state radius $R_{s s}$ of a freely growing spheroid as a function of adhesion strength $f_{1}^{*}$ for different growth forces $B^{*}$. The solid lines represent fits of $R_{s s}=\alpha /\left(f_{1}^{*}-f_{c}^{*}\right)$. All data were measured in full periodic boundaries. Error bars represent standard deviations. 
a)

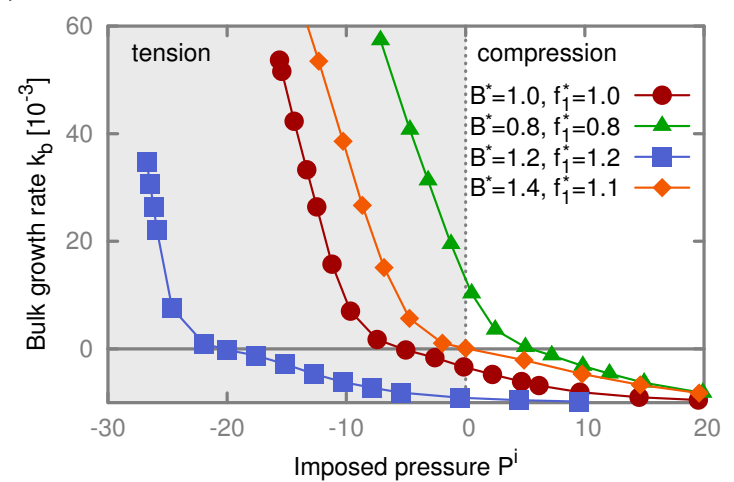

b)

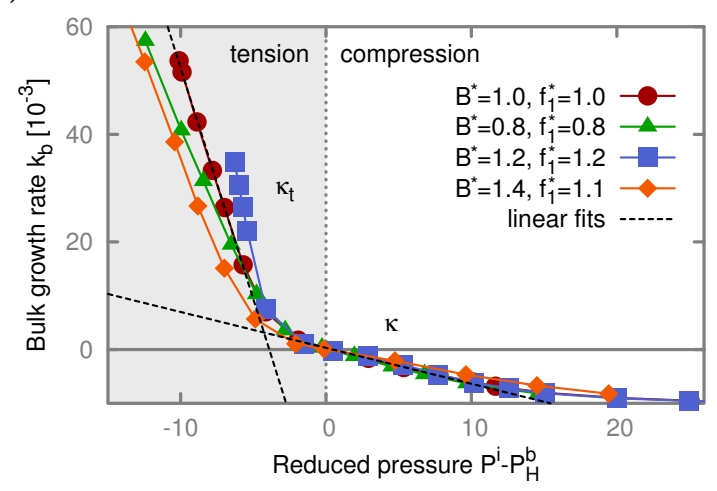

Fig. 4: Growth rate under compression and tension. a), bulk growth rate $k_{b}$ as a function of the imposed pressure $P^{i}$ for several parameter sets. b), same as in a) but shifted by the bulk homeostatic pressure $P_{H}^{b}$, which collapses all curves onto one. Note the two linear regimes.

of such a tissue spheroid reads:

$$
\partial_{t} R^{3}=k_{b} R^{3}+3 \lambda \delta k_{s} R^{2},
$$

where $k_{b}$ is the growth rate in bulk and $\delta k_{s}>0$ is the growth rate increment in a small region $\lambda$ at the surface. A stable steady state $\left(\partial_{t} R^{3}=0\right)$ can only arise if $k_{b}<0$ and $\delta k_{s}>-k_{b}$. An unstable steady state exists for $k_{b}>0$ and $\delta k_{s}<-k_{b}$. Plugging equation (1) into equation (4) leads to a steady state radius $R_{s s} \propto 1 / P_{H}^{b}$, which can be rewritten to $R_{s s} \propto 1 /\left(f_{1}^{*}-f_{c}^{*}\right)$ using our earlier simulation results, diverging at the same critical adhesion strength. This dependence is perfectly reproduced in the simulations as shown in Fig. 3.

As a next step, we analyzed the bulk growth rate $k_{b}$ for a wide range of imposed pressures $P^{i}$. Although experimentally not yet accessible, we simulated not only tissues under compression $\left(P^{i}>0\right)$ but also tissues under tension $\left(P^{i}<0\right)$. Our studies reveal a distinct non linearity of the bulk growth rate $k_{b}$ with the imposed pressure $P^{i}$.

In Fig. 4 the bulk growth rate is displayed as a function of the imposed pressure $P^{i}$ for different parameter sets. The general behavior separates into three distinct regimes. Equation (1) holds in the domain close to the a)

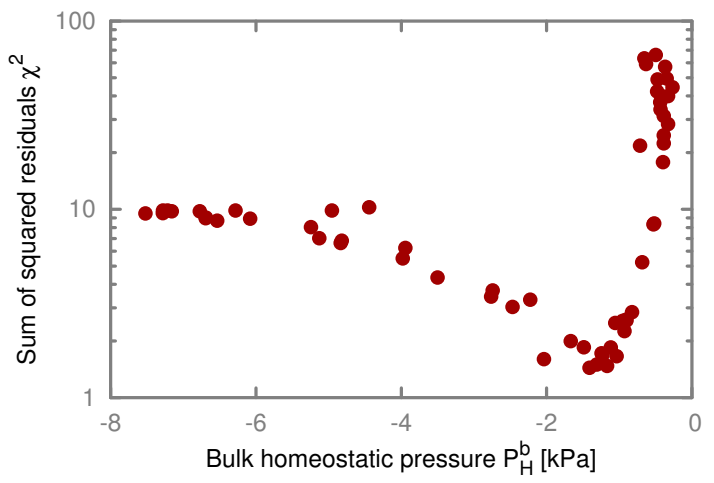

b)

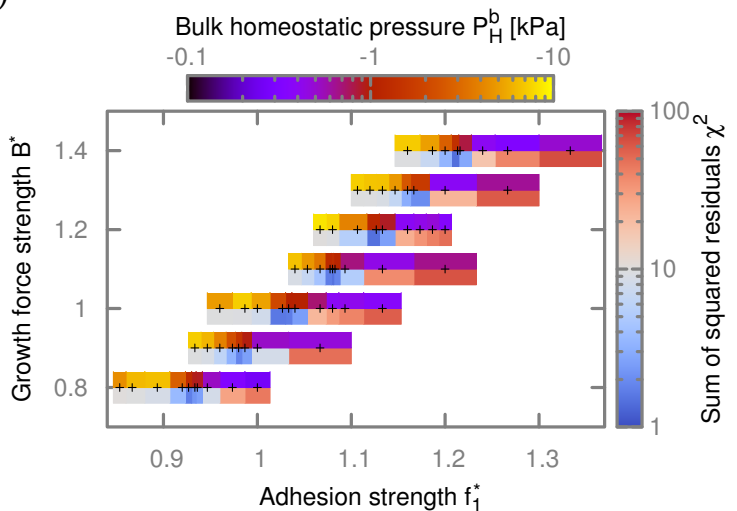

Fig. 5: Sum of squared residuals. a) The sum of squared residuals $\chi^{2}$ as a function of the bulk homeostatic pressure $P_{H}^{b}$. There exists a clear minimum around -1 to $-2 \mathrm{kPa}$. b) The sum of squared residuals $\chi^{2}$ as a function of growth force $B^{*}$ and adhesion strength $f_{1}^{*}$ (heat map directly below black crosses with scale on the right) and the bulk homeostatic pressure $P_{H}^{b}$ as a function of the same parameters (heat map above black crosses with scale above plot). The black crosses mark the exact values for $B^{*}$ and $f_{1}^{*}$ used in the according simulations. Note that the low $\chi^{2}$ (blue) always coincide with a $P_{H}^{b}$ of -1 to $-2 \mathrm{kPa}$ (red/orange).

homeostatic pressure (with slope $\kappa$ ), which extends a bit further towards cell compression. Under stronger tension, another linear regime with slope $\kappa_{t}$ is observed. The slopes $\kappa$ are very $\operatorname{similar}\left(\kappa \approx(-0.60 \pm 0.07) \cdot 10^{-3}\right)$ for all tested parameter sets, while $\kappa_{t}$ seems to change $\left(\kappa_{t} \approx(-8.0 \pm 1.9) \cdot 10^{-3}\right)$, however, not statistically significant. The third region reveals an asymptotic behavior, where cell division is mostly suppressed due to the imposed pressure and therefore the growth rate $k_{b}$ approaches the fixed apoptosis rate. All curves fall on top of each other, when we shift them by their bulk homeostatic pressure.

Former experiments measured the growth of cellular aggregates under pressure $[8,24]$. From the growth curves and the surface growth model of Eq. 4, the bulk growth rate and the surface rate increment have been extracted for 5 different cell lines under various intensities of pressure (see Tab. 1). All studied cell lines have a negative bulk growth rate at zero external pressure, indicative of a 
a)

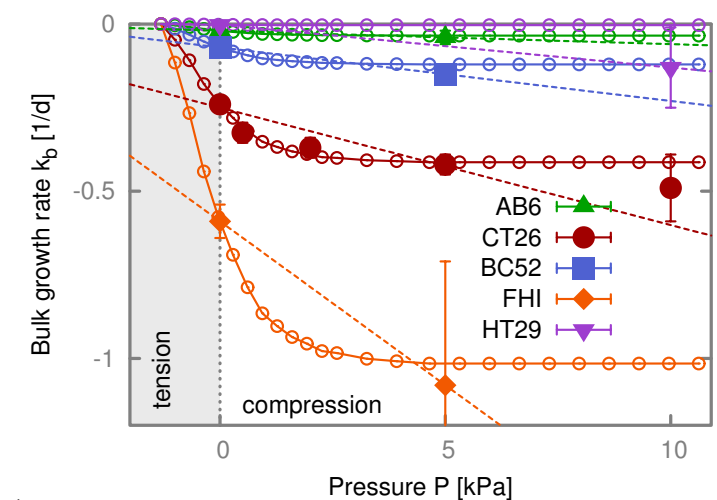

b)

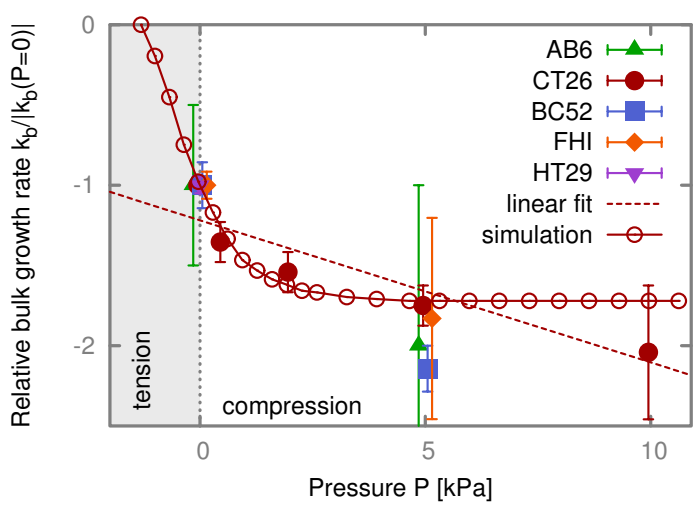

Fig. 6: Experimental data and simulation fits. a), experimentally determined absolute bulk growth rate $k_{b}$ as a function of the pressure $P$ for different cell lines (closed symbols). The open symbols show the same simulation data that was fitted to CT26, rescaled according to $k_{b}(0)$. The dashed lines represent a linear extrapolation of each cell line. Note that all cell lines have a negative growth rate at zero pressure. b), same as in a) but rescaled with the growth rate at zero pressure $k_{b}(0)$. The dashed line represents a linear fit of the data of CT26 and the open symbols show simulation data with $P_{H}^{b} \simeq-1.3 \mathrm{kPa}$. The relative bulk growth rate of HT29 under pressure is off scale $(-65)$. Error bars obtained from jackknife estimation.

negative homeostatic pressure.

To estimate its magnitude, we extrapolate the bulk growth rate linearly to zero, using Eq. 1 . This yields a negative bulk homeostatic pressure roughly around $-5 \mathrm{kPa}$. The only exception, for the HT29 cell line, results in a much smaller value of $-0.16 \mathrm{kPa}$ (see Tab. 1). However, simulations show that the linear extrapolation is not valid for all applied pressures, and the data of CT26 further underlines this non linear trend.

In order to match the simulations to the experimental data of CT26, we varied the parameters growth force $B^{*}$ and adhesion strengths $f_{1}^{*}$ around their standard values from [8] and measured the growth rate for several different imposed pressures. The pressure rescaling factor $\hat{P}$ was then determined for each parameter set by minimizing the
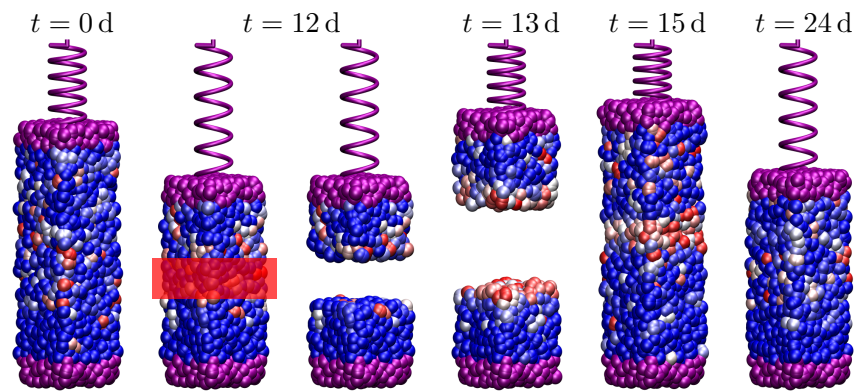

Fig. 7: Virtual 3d laser cut experiment. Set-up and colors are the same as in Fig. 1. The tissue grows, fills the compartment and pulls down the piston, until a steady state under tension is reached. Upon laser exposure (visualized by the red box), cells are taken out of the simulation and the piston relaxes back to its equilibrium position. The free surface increases the growth rate of the tissue until the wound is closed and the same steady state under tension emerges again.

sum of squared residuals

$$
\chi^{2}=\sum_{i}\left(k_{b}^{C T 26}\left(P_{i}\right)-k_{b}^{s i m}\left(P_{i} / \hat{P}\right)\right)^{2} .
$$

In Fig. 5a $\chi^{2}$ is plotted as a function of the resulting bulk homeostatic pressure $P_{H}^{b}$, with a clear minimum around -1 to $-2 \mathrm{kPa}$. Although we find a $f_{1}^{*}$ with a low $\chi^{2}$ for each $B^{*}$, the resulting homeostatic pressure always lies around -1 to $-2 \mathrm{kPa}$ (see Fig. $5 \mathrm{~b}$ ).

Surprisingly, a rescaling of the fitted simulations according to the bulk growth rate at zero pressure nicely reproduces the data of three of the remaining four cell lines (see Fig. 6a). Therefore, rescaling the experimental data with the zero pressure growth rate collapses all curves onto one (except HT29). This is shown in Fig. 6b and suggests a certain universality to the shape.

Conclusions. - In summary we showed that a negative homeostatic pressure is possible and stable. Indeed, such a tensile state is suggested by experimental data and fitting our simulations, estimates the homeostatic pressure of four out of five analyzed cell lines to be of the order of minus one to two $\mathrm{kPa}$. The homeostatic pressure grows with compressibility, growth force and the reduction of adhesion. It is known that as cancer evolves, tumor cells have a lower expression of the cell-cell adhesive protein Ecadherin [25]. Here, we show that a reduction of cell-cell adhesion could increase the homeostatic pressure, which would favor cancer progression [6]. Furthermore, the increase of homeostatic pressure with cell compressibility hints for an explanation why cancer cells across many different origins are consistently found to be softer [17-23].

The negative homeostatic pressure offers a novel and simple explanation of how tensile homeostasis is maintained. Indeed, many epithelia are found to be under tension in vivo $[2,3,26-28]$. Laser ablation experiments typically show recoil velocities after the cut, clearly prov- 
Table 1: Bulk growth rate $k_{b}$ of several different cell lines with and without pressure. The pressure $P_{\text {Dex }}$ exerted by the added Dextran is $10 \mathrm{kPa}$ for HT29 and CT26 and $5 \mathrm{kPa}$ otherwise. Furthermore, the bulk homeostatic pressure estimation via linear interpolation is shown. Growth rates taken from $[8,24]$.

\begin{tabular}{l|lll} 
& $k_{b}(0)\left[\mathrm{d}^{-1}\right]$ & $k_{b}\left(P_{\text {Dex }}\right)\left[\mathrm{d}^{-1}\right]$ & $P_{H}^{b}[\mathrm{kPa}]$ \\
\hline AB6 & $-0.02(1)$ & $-0.04(2)$ & $-5(7)$ \\
BC52 & $-0.07(1)$ & $-0.15(1)$ & $-4(1)$ \\
FHI & $-0.59(5)$ & $-1.08(37)$ & $-6(5)$ \\
HT29 & $-0.0020(1)$ & $-0.13(12)$ & $-0.16(15)$ \\
CT26 & $-0.24(1)$ & $-0.49(10)$ & $-14(4)$
\end{tabular}

ing the epithilia to be under tension. Intuitively one could think a tensile homeostasis is unstable: a simple cut relaxes stress, leading to an elevated pressure and thus more apoptosis. However, as we have shown, the surface growth effect stabilizes the tensile state. In the simulations we can perform a $3 \mathrm{~d}$ virtual laser cut. While a cut leads to a certain recoil, cells grow faster at the new free surface, closing the wound (see Fig. 7 and Supplementary Movie S2).

One question that remains unanswered is to what could be the evolutionary advantage of a tensile homeostatic state. While we can only speculate, a few ideas suggest themselves. The combination of surface growth and bulk death naturally lead to finite sized compartments. The mechanism described here could be a simple way of size control. From a mechanics point of view, a tensile tissue connected to a stiff skeleton seems more capable of sustaining its shape and integrity under constantly changing external forces.

\section{REFERENCES}

[1] Ingber D. E., Madri J. A. and Jamieson J. D., Proceedings of the National Academy of Sciences, 78 (1981) 3901.

[2] Wozniak M. A. and Chen C. S., Nature Reviews Molecular Cell Biology, 10 (2009) 34.

[3] Butcher D. T., Alliston T. and Weaver V. M., Nature Reviews Cancer, 9 (2009) 108.

[4] Drasdo D. and Hoenme S., New Journal of Physics, 14 (2012) 055025.

[5] Taloni A., Alemi A. A., Ciusani E., Sethna J. P., Zapperi S. and La Porta C. A. M., PLoS ONE, 9 (2014) e94229.

[6] Basan M., Risler T., Johnny J., SastreGarau X. and Prost J., HFSP Journal, 3 (2009) 265.

[7] Alessandri K., Sarangi B. R., Gurchenkov V. V., Sinha B., Kieling T. R., Fetler L., Rico F., Scheuring S., Lamaze C., Simon A., Geraldo S., Vignjevi D., Domjean H., Rolland L., Funfak A., Bibette J., Bremond N. and Nassoy P., Proceedings of the National Academy of Sciences, 110 (2013) 14843.

[8] Montel F., Delarue M., Elgeti J., Malaquin L., Basan M., Risler T., Cabane B., Vignjevic D., Prost J., Cappello G. and Joanny J.-F. M. C., Phys. Rev. Lett., 107 (2011) 188102.
[9] Montel F., Delarue M., Elgeti J., Vignjevic D., Cappello G. and Prost J., New Journal of Physics, 14 (2012) 055008.

[10] Hirschhaeuser F., Menne H., Dittfeld C., West J., Mueller-Klieser W. and Kunz-Schughart L. A., Journal of Biotechnology, 148 (2010) 3.

[11] Delarue M., Montel F., Caen O., Elgeti J., Siaugue J.-M., Vignjevic D., Prost J., JoAnny J.-F. M. C. and Cappello G., Phys. Rev. Lett., 110 (2013) 138103.

[12] Basan M., Prost J., Joanny J.-F. and Elgeti J., Physical Biology, 8 (2011) 026014.

[13] Allen M. P., Computer simulation of liquids (Clarendon Press Oxford University Press, Oxford England New York) 1987.

[14] Tsai D. H., The Journal of Chemical Physics, 70 (1979) 1375.

[15] Minc N., Boudaoud A. and Chang F., Current Biology, 19 (2009) 1096.

[16] Gonzalez-Rodriguez D., Bonnemay L., Elgeti J., Dufour S., Cuvelier D. and Brochard-Wyart F., Soft Matter, 9 (2013) 2282.

[17] Runge J., Reichert T., Fritsch A., Ks J., Bertolini J. and Remmerbach T., Oral Diseases, 20 (2014) e120.

[18] Jonas O., Mierke C. T. and Kas J. A., Soft Matter, 7 (2011) 11488.

[19] Fritsch A., Hockel M., Kiessling T., Nnetu K. D., Wetzel F., Zink M. and Kas J. A., Nature Physics, 6 (2010) 730 .

[20] Remmerbach T. W., Wottawah F., Dietrich J., LinCOLn B., Wittekind C. and GucK J., Cancer Research, 69 (2009) 1728.

[21] Cross S. E., Jin Y.-S., RaO J. and Gimzewski J. K., Nature Nanotechnology, 2 (2007) 780.

[22] Guck J., Schinkinger S., Lincoln B., Wottawah F., Ebert S., Romeyke M., Lenz D., Erickson H. M., Ananthakrishnan R., Mitchell D., Ks J., Ulvick S. and Bilby C., Biophysical Journal, 88 (2005) 3689.

[23] Lekka M., Laidler P., Gil D., Lekki J., Stachura Z. and Hrynkiewicz A. Z., European Biophysics Journal, 28 (1999) 312.

[24] Delarue M., Montel F., Vignjevic D., Prost J., JoAnNy J.-F. and CAPpello G., Biophysical Journal, 106 (2014) 42a.

[25] Weinberg R., The biology of cancer (Garland Science, New York) 2007.

[26] Trepat X., Wasserman M. R., Angelini T. E., Millet E., Weitz D. A., Butler J. P. and Fredberg J. J., Nature Physics, 5 (2009) 426.

[27] Heisenberg C.-P. and Bellache Y., Cell, 153 (2013) 948.

[28] Martin A. C. and Goldstein B., Development, 141 (2014) 1987. 